\title{
A SEARCH FOR OB ASSOCIATIONS NEAR SOUTHERN LONG-PERIOD CEPHEIDS. V. AQ PUPPIS AND V620 PUPPIS
}

\author{
D. G. Turner ${ }^{1,5}$, S. van den Bergh ${ }^{2,6}$, P. F. Younger ${ }^{2}$, D. J. Majaess ${ }^{1}$, M. H. Pedreros ${ }^{3,5}$, And L. N. Berdnikov ${ }^{4,7,8}$ \\ ${ }^{1}$ Department of Astronomy and Physics, Saint Mary's University, Halifax, NS B3H 3C3, Canada; turner@ap.smu.ca \\ ${ }^{2}$ Dominion Astrophysical Observatory, Herzberg Institute of Astrophysics, National Research Council of Canada, \\ 5071 West Saanich Road, Victoria, BC V8X 4M6, Canada \\ ${ }^{3}$ Departamento de Física, Facultad de Ciencias, Universidad de Tarapacá, Casilla 7-D, Arica, Chile \\ ${ }^{4}$ Moscow M. V. Lomonosov State University, Sternberg Astronomical Institute, Moscow, Russia \\ Received 2012 September 20; accepted 2012 October 12; published 2012 November 15
}

\begin{abstract}
A photometric $U B V$ survey is presented for 610 stars in a region surrounding the Cepheid AQ Puppis and centered southwest of the variable, based upon photoelectric measures for 14 stars and calibrated iris photometry of photographic plates of the field for 596 stars. An analysis of reddening and distance for program stars indicates that the major dust complex in this direction is $\sim 1.8 \mathrm{kpc}$ distant, producing differential extinction described by a ratio of total-to-selective extinction of $R=A_{V} / E_{B-V}=3.10 \pm 0.20$. Zero-age main-sequence fitting for the main group of B-type stars along the line of sight yields a distance of $3.21 \pm 0.19 \mathrm{kpc}\left(V_{0}-M_{V}=12.53 \pm 0.13\right.$ s.e. $)$. The 29.97 Cepheid AQ Pup, of field reddening $E_{B-V}=0.47 \pm 0.07\left(E_{B-V}(\mathrm{~B} 0)=0.51 \pm 0.07\right)$, appears to be associated with B-type stars lying within $5^{\prime}$ of it as well as with a sparse group of stars, designated Turner 14, centered south of it at J2000.0 $=07: 58: 37,-29: 25: 00$, with a mean reddening of $E_{B-V}=0.81 \pm 0.01$. AQ Pup has an inferred luminosity as a cluster member of $\left\langle M_{V}\right\rangle=-5.40 \pm 0.25$ and an evolutionary age of $3 \times 10^{7}$ yr. Its observed rate of period increase of $+300.1 \pm 1.2 \mathrm{~s} \mathrm{yr}^{-1}$ is an order of magnitude larger than what is observed for Cepheids of comparable period in the third crossing of the instability strip, and may be indicative of a high rate of mass loss or a putative fifth crossing. Another sparse cluster, designated Turner 13, surrounds the newly recognized 2. 59 Cepheid V620 Pup, of space reddening $E_{B-V}=0.64 \pm 0.02\left(E_{B-V}(\mathrm{~B} 0)=0.68 \pm 0.02\right)$, distance $2.88 \pm 0.11 \mathrm{kpc}\left(V_{0}-M_{V}=12.30 \pm 0.08\right.$ s.e. $)$, evolutionary age $10^{8} \mathrm{yr}$, and an inferred luminosity as a likely cluster member of $\left\langle M_{V}\right\rangle=-2.74 \pm 0.11$. V620 Pup is tentatively identified as a first crosser, pending additional observations.
\end{abstract}

Key words: open clusters and associations: general - stars: fundamental parameters - stars: variables: Cepheids

Online-only material: machine-readable and VO tables

\section{INTRODUCTION}

The most important Galactic calibrators for the Cepheid period-luminosity (PL) relation are long-period pulsators, which are less frequently found in open clusters than their shortperiod cousins (e.g., Turner \& Burke 2002; Turner 2010). Such objects are massive and young enough, however, to belong to older portions of $\mathrm{OB}$ associations, which can often be delineated by photometric or spectroscopic methods. That philosophy initiated a program by Sidney van den Bergh 30 years ago to identify associated young B-type stars in the vicinity of bright southern hemisphere long-period Cepheids (van den Bergh et al. 1982, 1983, 1985; Turner et al. 1993), with offshoots involving studies of potential coincidences of long-period Cepheids with open clusters (Turner et al. 2005, 2009a). The present study involves the 29.97 Cepheid AQ Puppis $\left(\ell=246^{\circ} .1562, b=+0.1061\right)$, which presents unique complications arising from the high degree of differential reddening by interstellar dust along its line of sight.

\footnotetext{
5 Visiting Astronomer, Helen Sawyer Hogg Telescope, University of Toronto.

6 Visiting Astronomer, Cerro Tololo Inter-American Observatory, National

Optical Astronomy Observatory, which is operated by the Association of

Universities for Research in Astronomy (AURA), Inc., under cooperative agreement with the National Science Foundation.

7 Visiting Astronomer, Harvard College Observatory Photographic Plate Stacks, Cambridge, MA, USA.

8 Also at Isaac Newton Institute of Chile, Moscow Branch, Moscow, Russia.
}

The rediscovery of Cepheids in Galactic open clusters by Irwin in 1955 (Irwin 1955, 1958) was accompanied by additional, independent searches for Cepheid-cluster coincidences (Kholopov 1956; Kraft 1957; van den Bergh 1957; Tifft 1959). A later study by Tsarevsky et al. (1966) with an updated cluster database revealed the spatial coincidence of AQ Pup with the coronal region of the cluster Ruprecht 43 , but without further follow-up, possibly because of an uncertain nature for the cluster. A possible association of AQ Pup with Pup OB2 at $d \simeq 4.4 \mathrm{kpc}$ was studied by Fernie et al. (1966), but without definitive conclusions. Grubissich (1968) took a more positive view of the same data while revising the distance of Pup OB2 to $2.9 \mathrm{kpc}$, subsequently confirmed by Tsarevsky (1970). An alternate possibility for an association of AQ Pup with Pup OB1 at $d \simeq 2.5 \mathrm{kpc}$ was suggested by Havlen (1972), although Turner (1981) argued that the Cepheid appeared unlikely to be a member of either association.

Star counts in the immediate vicinity of the Cepheid did reveal a slight density enhancement (Turner; see Evans \& Udalski 1994), suggesting the possibility that the region near AQ Pup might contain the sparse remains of an uncataloged open cluster or association, now designated as Turner 12 (Dias et al. 2002; B. S. Alessi 2012, private communication). A preliminary assessment (Turner \& Burke 2002) designated the group as Pup OB3, for lack of a more definitive term. The present photometric survey of the field explores the preliminary findings further to reveal the possible open cluster connection that exists. 
Subsequent to the original data collection and measurement an additional Cepheid was found to lie in the survey field, the 2.59 Cepheid V620 Puppis $(\ell=246.3115, b=-0.1264$; Kazarovets et al. 2008), originally NSV 03832 but recognized as a classical Cepheid from the ASAS-3 survey (Pojmanski 2002). By happy circumstance, V620 Pup appears to lie in a previously unrecognized sparse open cluster, so is itself a potential calibrator for the short-period end of the PL relation. The present study also discusses the independent case for its cluster membership.

There is another long-period Cepheid in the region of AQ Pup, namely, the 14.15 variable LS Pup. However, it falls just west of the surveyed region, so is not discussed here.

This is the final study in a series that was initially based on photographic photometry tied to skeleton photoelectric sequences. It has been a very large project that has by necessity extended over a number of decades. For the sake of homogeneity it was necessary to use the same techniques (e.g., iris photometry) for all program fields, although there have been improvements to the original methodology, such as incorporating additional photoelectric standards, refining the iris photometry techniques to improve the precision of the results, and completing more comprehensive analyses of interstellar reddening, particularly differential reddening, which has been ubiquitous in all survey fields.

The introduction of CCD detectors in the intervening years has changed the nature of photometric surveys. Greater precision could be achieved at present through use of a CCD detector, although mosaicing would be needed to cover the fields studied. Accurate corrections for the effects of interstellar reddening are also best achieved using $U B V$ photometry, which can be a challenge to achieve with the panchromatic response of most CCD devices (see Massey 2002). In the end what counts is the result obtained, not the technique used, as the present study demonstrates.

\section{OBSERVATIONAL DATA}

The data for the present study include photoelectric $U B V$ photometry of 14 stars in the field of AQ Pup from observations obtained with the Cerro Tololo $1.5 \mathrm{~m}$ telescope in 1979 March and the University of Toronto's Helen Sawyer Hogg $0.6 \mathrm{~m}$ telescope in 1976 March and 1987 January, when it was located on Cerro Las Campanas, Chile. Details are provided by Turner (1981), van den Bergh et al. (1982), Shorlin et al. (2004), and Turner et al. (2009a). The data for the 14 stars used as photoelectric standards are summarized in Table 1, where the stars are identified by Arabic letters and their co-ordinates in the 2MASS catalog (Cutri et al. 2003). Three of the stars are numbered in the Catalogue of Luminous Stars in the Southern Milky Way (Stephenson \& Sanduleak 1971).

The photoelectric data were supplemented by photographic $U B V$ photometry for 596 stars in the vicinity of AQ Pup obtained from iris photometry of plates in $U, B$, and $V$ taken with the $0.9 \mathrm{~m}$ Swope telescope on Cerro Las Campanas in 1978 May and 1982 February (two plates in $B$ and one plate in each of $V$ and $U$ ). The iris measures were obtained using the Cuffey Iris Astrophotometer at Saint Mary's University along with the prescriptive techniques described by Turner \& Welch (1989), which are designed to generate data with a precision approaching $\pm 0.03 \mathrm{mag}$. The resulting photographic measures encompass a field of $\sim 18^{\prime}$ radius centered on J2000.0 coordinates 07:57:30.696, -29:17:54.79 (Figure 1) and are summarized in Table 2.

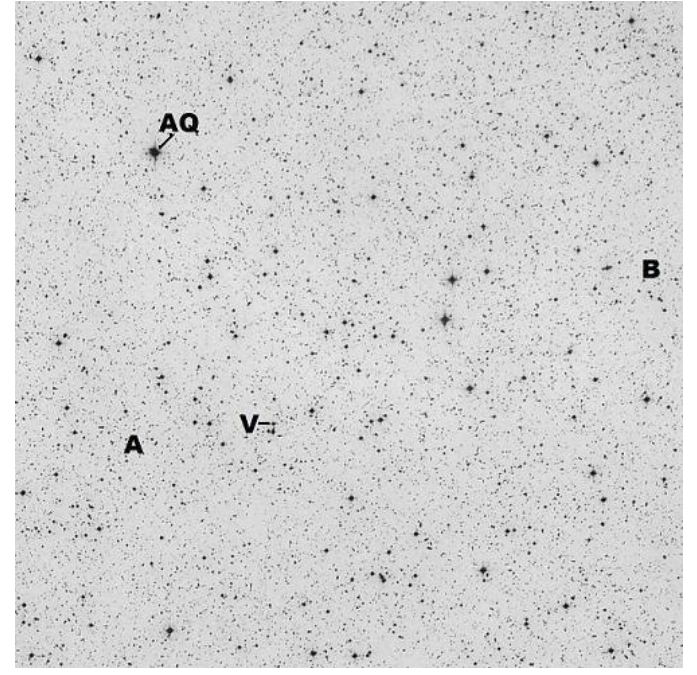

Figure 1. Chart (north up, east left) for the $38^{\prime}$ diameter field near AQ Pup (AQ) and V620 Pup (V) centered on J2000 = 07:57:30.7, -29:17:54.8, derived from the red ESO-SRC image. The regions designated as Fields A and B in the text are denoted by the letters $\mathrm{A}$ and $\mathrm{B}$.

Table 1

Photoelectric Data for the AQ Pup Field

\begin{tabular}{lcccccr}
\hline \hline Star & R.A.(2000) & Decl.(2000) & $V$ & $B-V$ & $U-B$ & $n$ \\
\hline AQa & $07: 58: 22.442$ & $-29: 07: 39.57$ & 11.13 & 1.33 & +1.45 & 10 \\
AQb & $07: 58: 23.054$ & $-29: 07: 37.61$ & 12.74 & 0.21 & +0.14 & 10 \\
A & $07: 58: 24.378$ & $-29: 07: 52.60$ & 14.70 & 0.60 & +0.26 & 1 \\
B & $07: 58: 09.256$ & $-29: 09: 47.08$ & 10.23 & 0.36 & +0.13 & 3 \\
C & $07: 58: 20.117$ & $-29: 10: 15.86$ & 12.50 & 0.53 & -0.29 & 3 \\
D & $07: 58: 20.818$ & $-29: 09: 53.60$ & 14.45 & 0.63 & +0.18 & 1 \\
E & $07: 58: 22.950$ & $-29: 10: 19.76$ & 13.49 & 0.50 & +0.28 & 3 \\
F & $07: 58: 19.870$ & $-29: 11: 15.82$ & 11.58 & 0.49 & -0.34 & 3 \\
G & $07: 58: 18.654$ & $-29: 11: 59.54$ & 13.01 & 0.24 & +0.19 & 3 \\
H & $07: 58: 21.097$ & $-29: 05: 57.74$ & 13.31 & 0.62 & +0.13 & 3 \\
I & $07: 58: 24.257$ & $-29: 06: 36.08$ & 14.90 & 0.53 & +0.41 & 1 \\
J $^{\mathrm{a}}$ & $07: 57: 56.655$ & $-29: 05: 47.47$ & 11.63 & 0.19 & -0.59 & 3 \\
K $^{\mathrm{b}}$ & $07: 57: 25.109$ & $-29: 10: 03.75$ & 9.79 & 0.20 & -0.70 & 8 \\
L $^{\mathrm{c}}$ & $07: 56: 26.407$ & $-29: 25: 26.13$ & 10.28 & 0.43 & -0.44 & 5 \\
\hline
\end{tabular}

Notes.

a LSS 890.

b LSS 888 .

c LSS 876.

Table 2

Photographic Data for the AQ Pup Field

\begin{tabular}{lcccrr}
\hline \hline R.A.(2000) & Decl.(2000) & $V$ & $B-V$ & $U-B$ & Comments \\
\hline 07:56:06.216 & $-29: 14: 20.10$ & 12.78 & 0.67 & -0.46 & \\
07:56:06.556 & $-29: 17: 36.63$ & 12.88 & 1.67 & 1.17 & \\
07:56:07.208 & $-29: 21: 34.57$ & 12.29 & 0.78 & 0.07 & \\
07:56:07.554 & $-29: 20: 15.90$ & 13.55 & 0.49 & -0.10 & \\
07:56:08.866 & $-29: 16: 05.84$ & 11.97 & 0.71 & -0.13 & \\
07:56:09.486 & $-29: 21: 04.73$ & 13.17 & 0.44 & 0.38 & \\
$07: 56: 09.679$ & $-29: 15: 34.22$ & 13.29 & 0.66 & 0.60 & \\
$07: 56: 10.370$ & $-29: 15: 27.01$ & 12.30 & 1.03 & 0.65 & \\
$07: 56: 12.414$ & $-29: 15: 20.09$ & 12.71 & 0.55 & -0.07 & \\
$07: 56: 12.870$ & $-29: 15: 05.31$ & 12.81 & 0.56 & -0.26 & \\
\hline
\end{tabular}

(This table is available in its entirety in machine-readable and Virtual Observatory (VO) forms in the online journal. A portion is shown here for guidance regarding its form and content.) 


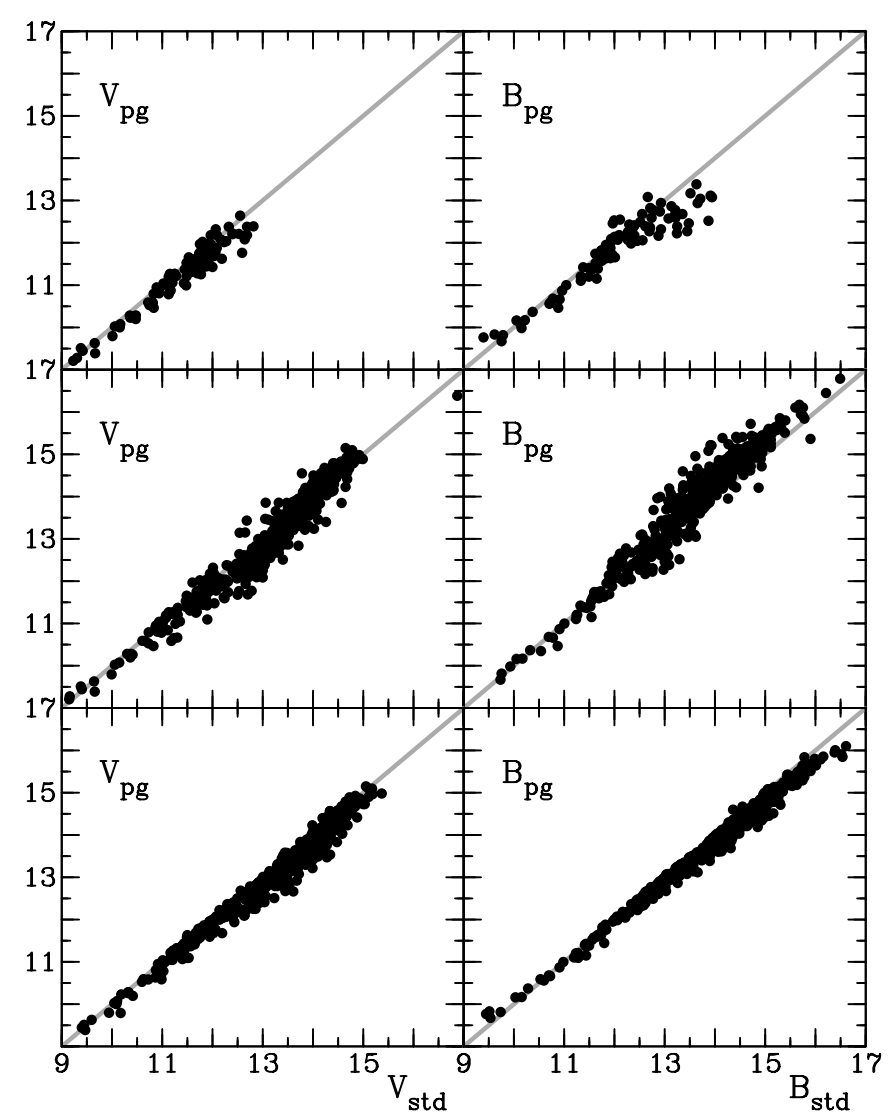

Figure 2. Comparison of the present photographic $V$ (left) and $B$ (right) magnitudes with those measured by ESA (1997; top), NOMAD (Zacharias et al. 2005; middle), and the AAVSO's APASS program (bottom). Gray lines denote the correlation expected for perfect agreement.

The effectiveness of surveys such as this varies in direct proportion to the accuracy and precision of the input data. The precision is limited by photon counting statistics for the photoelectric observations and by photographic grain noise for the photographic photometry, and in our experience is typically \pm 0.01 and \pm 0.03 to \pm 0.04 , respectively, in both magnitudes and colors. The techniques adopted by Turner \& Welch (1989) have been demonstrated to reach such a level of precision for iris measures of photographic plates, provided that steps are taken to measure complete stellar images, including extended tails, and which lead to magnitude calibrations that are simple power laws of the iris readings. That was the case here. The accuracy of the observations is generally tested by comparison with the results of other studies for stars in common and by direct examination of the data, as illustrated here.

There is no other source of $U B V$ photometry available for the general field of AQ Pup, but recent surveys have generated $B V$ photometry for most program stars. A comparison of our photometry (Figure 2) with that of ESA (1997) from the Hipparcos and Tycho Catalogues reveals no obvious discrepancies. The photographic $V$ magnitudes agree closely with the ESA (1997) results for stars brighter than $V=11$ and stars of $11<V<12$, although the scatter is larger for the latter, which are near the limit for ESA photometry. The photographic $B$ magnitudes also agree closely with the ESA (1997) results for stars brighter than $B=12$ and stars of $12<B<12.6$, for which the scatter is larger. There are systematic trends in both $V$ and $B$ for fainter stars, which are probably beyond the true limits for ESA photometry. A similar result was found in a

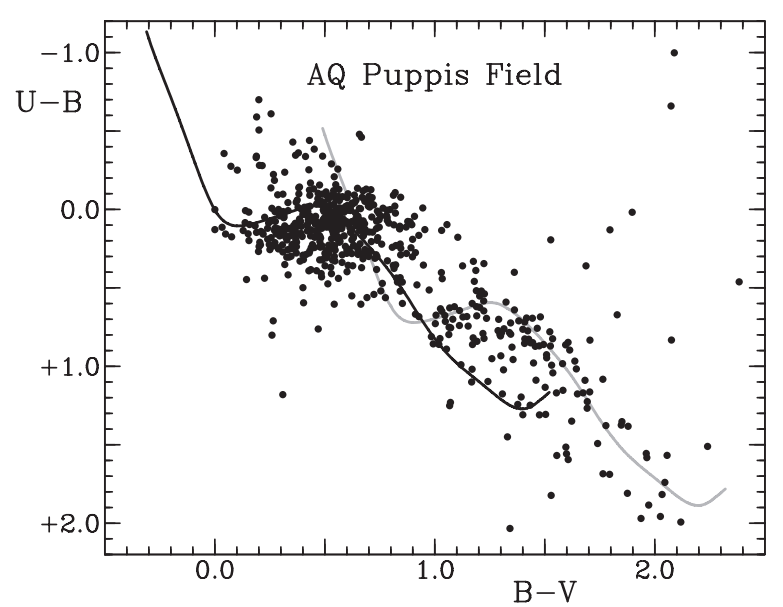

Figure 3. $U B V$ color-color diagram for measured stars in the AQ Pup field. The intrinsic relation for main-sequence stars is plotted as a solid black curve. The same relation reddened by $E_{B-V}=0.8$ is depicted by a gray curve.

comparison of the photographic photometry with that of Kharchenko \& Roeser (2001), which represents a tweaking of the ESA (1997) photometry.

The NOMAD survey resulting from calibrated scans of the POSS (Zacharias et al. 2005) is similar in some respects to our photographic survey, but reveals differences for stars fainter than $V=B=11$ (Figure 2) that are in opposite senses. Such deviations may reflect differences in the standard stars adopted for calibration purposes.

The APASS survey of the American Association of Variable Star Observers (AAVSO) contains CCD $B V$ photometry for most stars in our field. A comparison (Figure 2) of $V$ magnitudes for stars in common shows good agreement to $V \simeq 15$, but there are trends for many stars fainter than $V=12$, which appear to be measured systematically fainter by APASS. A comparison of the $B$ magnitudes is similar. There is generally good agreement for stars brighter than $B=12$, but systematic trends for most stars fainter than that, again with the stars being measured fainter by APASS. Both trends are the same as those seen in ESA (1997) photometry for stars at the photometric limits of that survey, which suggests potential problems with the calibration of the APASS results. Of course, there is also a potential for slight nonlinearity in the faint star calibration of the iris measures, but it is not clear how serious that may be.

Alternatively, the data themselves provide a good impression of their overall accuracy. Figure 3 is a $U B V$ color-color diagram for the complete sample of measured stars. Evident here is a selection of perhaps 10 stars that have questionable colors, some of which are stars in Table 2 for which nearby companions have affected the photometry. Others may lie near the survey limits, where the photometric calibrations are uncertain. But the majority of stars in Figure 2 display the colors expected for a large sample of stars affected by significant amounts of differential reddening. The bluest stars in the sample tend to be reddened stars with intrinsic colors of $(B-V)_{0}=-0.25$, corresponding to main-sequence spectral type B2 V, implying that the Puppis OB associations contain very few members in this section of the constellation. What is striking is the selection of $\sim 50$ stars that fall almost exactly on the intrinsic relation for unreddened main-sequence stars, and a similar group that lies extremely close to the intrinsic relation for AFGK-dwarfs reddened by $E_{B-V}=0.8$. If the data were affected by systematic magnitude-dependent errors, such features would simply not 


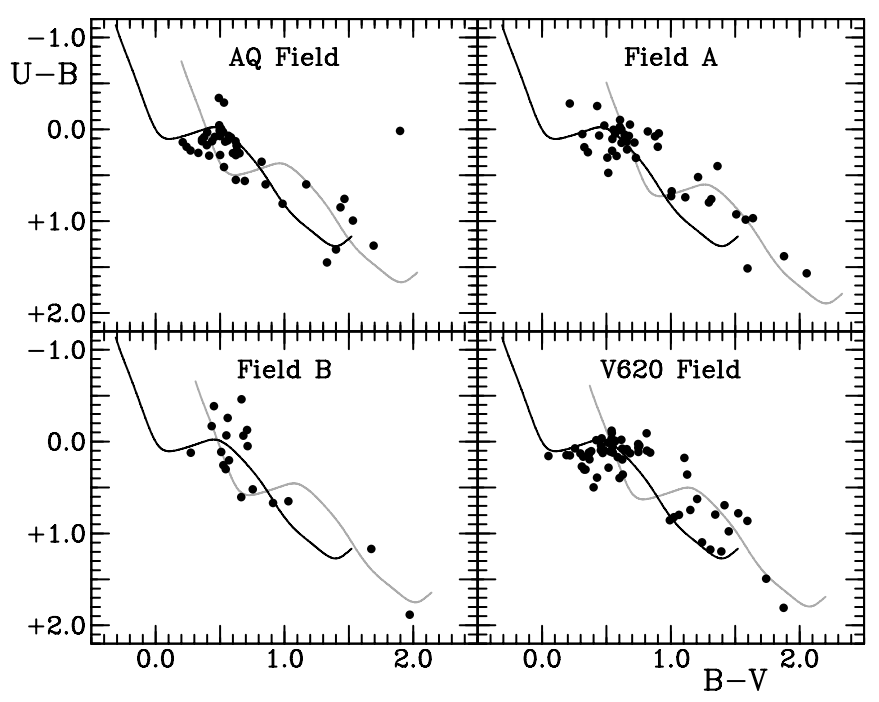

Figure 4. $U B V$ color-color diagrams for separate $5^{\prime}$ radius subfields in the AQ Pup region: surrounding AQ Pup (upper left), sparse cluster A centered at J2000.0 = 07:58:37, -29:25:00 (upper right), a group of stars (Field B) of common reddening centered at $\mathbf{J} 2000.0=07: 56: 06,-29: 13: 27$ (lower left), and the environs of V620 Pup (lower right). The plotted relations are those of Figure 3, except the color excesses are $E_{B-V}=0.51,0.81,0.62$, and 0.68, respectively.

occur. Of course, there is scatter in the observations, but not more than expected for the cited precision.

Further evidence regarding the general accuracy of the photometric observations is provided by isolating stars according to location in the field. Figure 4 contains individual color-color diagrams for four separate regions, of $5^{\prime}$ radius, in the survey area: AQ Pup (J2000.0 = 07:58:22, -29:07:48), Field A $(\mathrm{J} 2000.0=07: 58: 37,-29: 25: 00)$, Field B $(\mathrm{J} 2000.0=07: 56: 06$, -29:13:27), and V620 Pup (J2000.0 = 07:57:50, -29:23:03). Although differential reddening is present here as well, it is possible to identify small groups of stars of identical reddening, or even of little to no reddening. That also occurs in space reddening plots for the sample, where adjacent stars share similar reddenings, or zero reddening, to within \pm 0.01 to \pm 0.02 in $E_{B-V}$. Such results are only possible with photometry of reasonably high precision and accuracy. It implies good results for the derived space reddenings of the two Cepheids AQ Pup and V620 Pup, provided that differential reddening in their fields is not severe. The high degree of differential reddening in the field is also evident from the uncorrected color-magnitude diagram for program stars plotted in Figure 5. The scatter here is typical of fields where the color excesses $E_{B-V}$ for group stars exhibit a spread of a magnitude or more (Turner 1976b).

\section{ANALYSIS}

The $U B V$ data of Tables 1 and 2 plotted in Figure 3 were corrected for reddening using a reddening law for the field found previously (Turner 1981, 1989). It is described by $E_{U-B} / E_{B-V}=0.75+0.02 E_{B-V} . J H K_{s}$ observations for the same stars from the 2MASS catalog (Cutri et al. 2003) served as a guide for resolving ambiguities in likely intrinsic color for some stars, although excessive scatter in the $J H K_{s}$ data (Figure 6) actually introduced ambiguities of their own in many cases. The derived color excesses, $E_{B-V}$, were also normalized to those appropriate for a B0 star observed through the same amount of dust (Fernie 1963). Absolute magnitudes appropriate for zero-age main sequence (ZAMS) stars of the same intrinsic

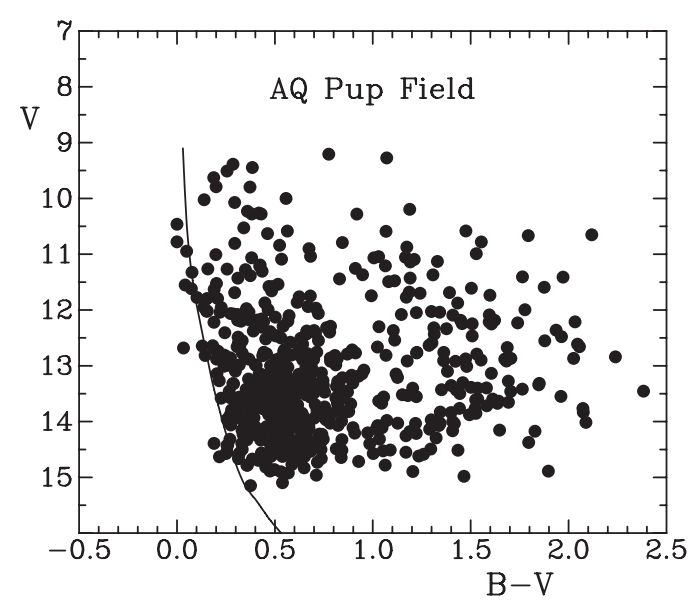

Figure 5. $B V$ color-magnitude diagram for measured stars in the AQ Pup field. The zero-age main sequence (ZAMS, black curve) is plotted for a reddening of $E_{B-V}=0.35$ and $V-M_{V}=13.6\left(V_{0}-M_{V}=12.55\right)$.

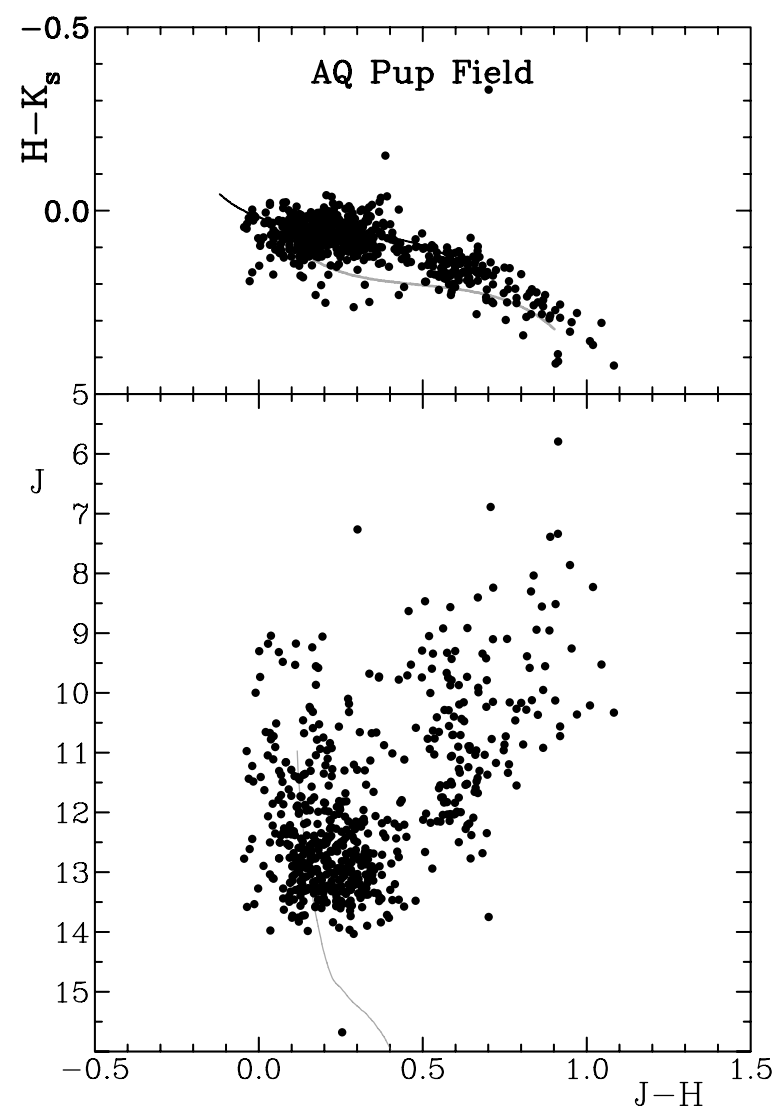

Figure 6. 2MASS color-color diagram (upper) and color-magnitude diagram (lower) for measured stars in the AQ Pup field. The intrinsic relation is plotted as a black curve, while a gray curve represents the same relation for a reddening equivalent to that used in Figure 3. The plotted ZAMS relation (gray curve, lower) has the same reddening for the intrinsic distance modulus of Figure 7.

color (Turner 1976a, 1979) were adopted in order to provide data suitable for a variable-extinction analysis of the stars (see Turner 1976a, 1976b). The results are presented in Figure 7.

A small proportion of stars in the AQ Pup field are unreddened late-type stars; none are O-type stars. However, there are many B-type, A-type, and F-type stars of various reddenings present throughout the region. Unreddened stars in the sample can be detected to intrinsic distance moduli reaching values as large as $V_{0}-M_{V}=11.25$, corresponding to distances of $1.78 \mathrm{kpc}$. 


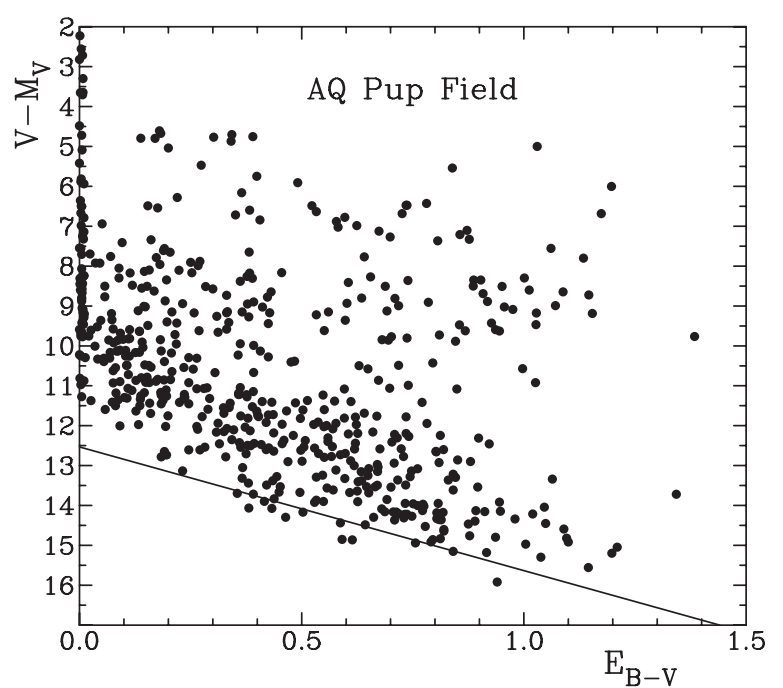

Figure 7. Variable-extinction diagram (plot of apparent distance modulus vs. color excess) for measured stars in the AQ Pup field, with absolute magnitudes assuming ZAMS luminosities. The line fitted to the lower envelope of the data has slope $R=3.10 \pm 0.20$ and zero-point $V_{0}-M_{V}=12.53 \pm 0.13$.

That is consistent with what was found previously by Havlen (1972) and Neckel \& Klare (1980) for distances to the dust clouds in this direction. It appears that the Galactic plane along the line of sight is relatively dust free for about $1.8 \mathrm{kpc}$, beyond which varied and occasionally substantial extinction arises.

The small density enhancement of stars denoted as Turner 12 at the center of the survey field of Figure 1 was originally detected by star counts using the low plate scale Vehrenberg Atlas (Vehrenberg 1964), which is superior to the POSS for detecting extended clusters. Most of the stars in that region have small color excesses relative to stars lying in the outer portions, and there appears to be a dust ring surrounding them on the north side that contains no program stars at all. The small density enhancement referred to as Turner 12 is therefore primarily an optical effect arising from patchy extinction in the field, and there is no clear photometric evidence for its existence as an extended star cluster or association.

The general trends in the data of Figure 7 appear to follow a value for the ratio of total-to-selective extinction, $R=$ $A_{V} / E_{B-V}$, that is close to 3 . Yet the distribution of data suggests that the reddening out to $1.8 \mathrm{kpc}$ may also be patchy. Note, for example, the sequences of points that clump toward intrinsic distance moduli of about 5 and 8, i.e., $100 \mathrm{pc}$ and $400 \mathrm{pc}$. If the stars are post main-sequence objects rather than dwarfs, then they must have luminosities of bright giants or supergiants if reddened by extinction arising in the main dust complex $1.8 \mathrm{kpc}$ distant.

There is a fairly distinct lower envelope in Figure 7 of reddened stars, presumably ZAMS stars, that have reddenings typically in excess of $E_{B-V}=0.35$. Least squares and non-parametric techniques applied to that group yield bestfitting values of $R=A_{V} / E_{B-V}=3.10 \pm 0.20$ s.e. and $V_{0}-M_{V}=12.53 \pm 0.13$ s.e., corresponding to a distance of $3.21 \pm 0.19 \mathrm{kpc}$. The value of $R$ is consistent with expectations for dust producing a reddening slope of $E_{U-B} / E_{B-V} \simeq 0.75$ (Turner 1994, 1996), even though the dust is not local.

Unreddened parameters for the group of stars surrounding V620 Pup are depicted in Figure 8 for an intrinsic distance modulus of $V_{0}-M_{V}=12.30 \pm 0.08$ derived for six stars that appear to lie on the ZAMS. There is a reasonably good fit

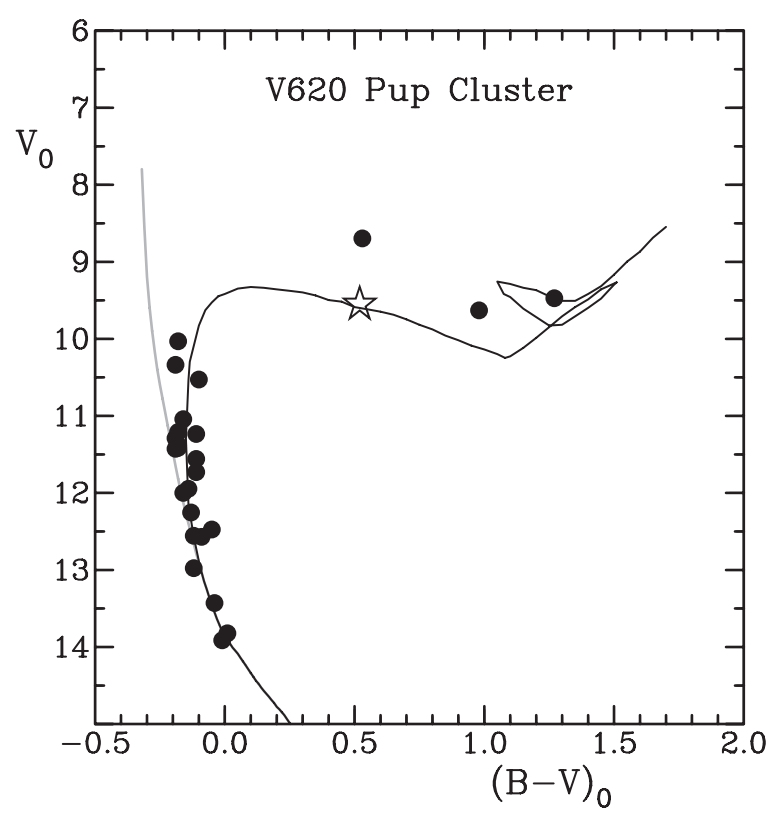

Figure 8. Reddening-corrected color-magnitude diagram for stars in the putative cluster surrounding V620 Pup. A gray curve represents the ZAMS for $V_{0}-M_{V}=12.30 \pm 0.08$, a black curve represents an isochrone for $\log t=8.0$, and the star symbol represents the mean parameters of V620 Pup.

of the data to a model isochrone for $\log t=8.0$ taken from Meynet et al. (1993). The implied distance of $2.88 \pm 0.11 \mathrm{kpc}$ requires confirmation from a deeper survey, and might be too small. The reality of the cluster, designated here as Turner 13, also needs to be confirmed by star counts and radial velocities. The field immediately surrounding V620 Pup does appear to contain an above average number of faint, reddened, blue stars, despite the relatively high reddening for the cluster, and that and the data of Figure 8 are presently the only evidence for the cluster's existence. Two of the red stars near the Cepheid may be red giant (GK-type) members of the cluster. They fit the $\log t=8.0$ isochrone reasonably well. The mean reddening of stars lying close to V620 Pup is $E_{B-V}(\mathrm{~B} 0)=0.68 \pm 0.02$ s.e., which corresponds to a field reddening for the Cepheid of $E_{B-V}=0.64 \pm 0.02$. The corresponding evolutionary age of Turner 13 and V620 Pup is $\sim 10^{8} \mathrm{yr}$.

Unreddened parameters for the group of stars labeled as Field A in Figure 3 are depicted in Figure 9 for the distance modulus derived in Figure 7. Although differential reddening is very clearly present in the region of this group of stars, designated here as Turner 14, the mean reddening of stars near the cluster core is $E_{B-V}(\mathrm{~B} 0)=0.81 \pm 0.01$ s.e. The reality of the cluster is indicated by an increase in the density of faint blue stars in the core regions of Turner 14, despite a rather large reddening. The identification of possible cluster members on the basis solely of reddening and location in the color-magnitude diagram of Figure 9 is difficult. The large apparent redward spread in the evolved portion of the cluster main sequence is an effect seen in other intermediate-age clusters (see Turner et al. 1992; Turner 1993) and may have a similar explanation in terms of circumstellar reddening for stars of large rotational velocity.

Additional members of Turner 14 can be found in the region surrounding AQ Pup, as shown in Figure 9. The identification of several of the stars as potential cluster members is motivated by the possibility that rapid rotation is inherent to many stars on the cluster main sequence. If that is incorrect, then six to seven of the stars could be removed from the sample as likely foreground 


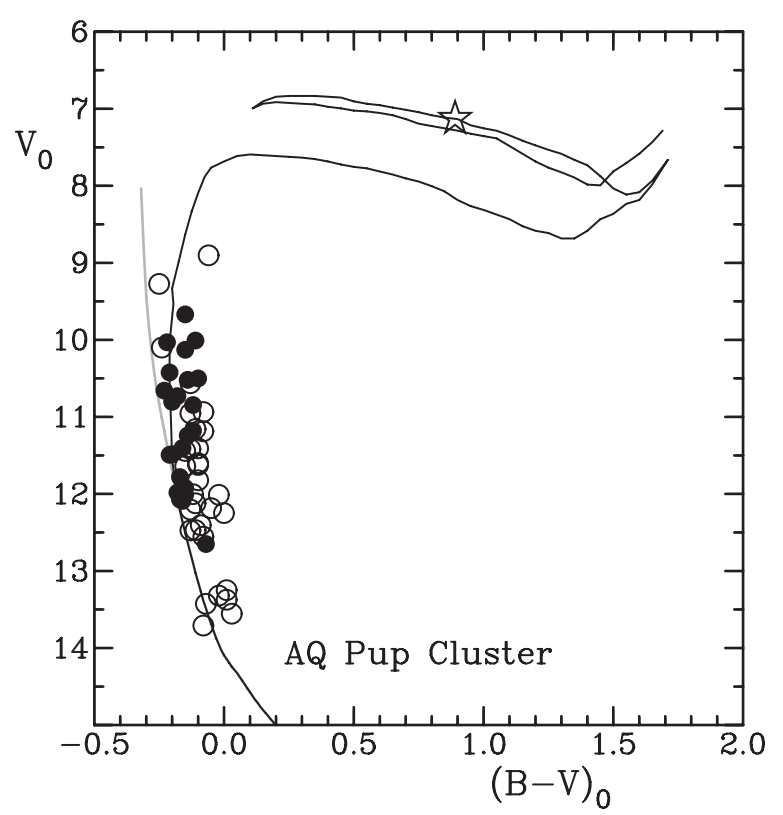

Figure 9. Reddening-corrected color-magnitude diagram for stars in the putative cluster south of AQ Pup (filled circles) and for stars surrounding the Cepheid (open circles). A gray curve represents the ZAMS for $V_{0}-M_{V}=$ $12.53 \pm 0.13$, a black curve represents an isochrone for $\log t=7.5$, and the star symbol represents the mean parameters of AQ Pup.

dwarfs. As noted in Figure 4, the mean reddening of stars lying within $2^{\prime}$ of AQ Pup is $E_{B-V}(\mathrm{~B} 0)=0.51 \pm 0.07$ s.e., equivalent to a space reddening for the Cepheid of $E_{B-V}=0.47 \pm 0.07$. Differential reddening is quite strong near the Cepheid and accounts for the large uncertainty in the results. The data for possible cluster members identified in Figure 9 provide a reasonably good fit to a model isochrone for $\log t=7.5$ taken from Meynet et al. (1993). The corresponding age of the cluster is $\sim 3 \times 10^{7} \mathrm{yr}$.

\section{AQ PUPPIS AND V620 PUPPIS}

In a simulation of period changes for long-period Cepheids tied to stellar evolutionary models, Pietrukowicz (2003) argues, from only five observed times of light maximum, that the period of AQ Pup does not appear to be changing. Such a conclusion emphasizes the importance of studying Cepheid period changes observationally using lengthy and rich temporal samples of light curve data.

Period changes for AQ Pup were established here from the examination of archival photographic plates of the variable in the collection of the Harvard College Observatory, as well as from an analysis of new and existing photometry for the star. A working ephemeris for AQ Pup based upon the available data was

$$
\mathrm{JD}_{\max }=2435156.13+29.985704 E \text {, }
$$

where $E$ is the number of elapsed cycles. An extensive analysis of all available observations produced the data summarized in Table 3, which lists results for 79 different epochs of light maximum derived from the complete light curves using Hertzsprung's method (Berdnikov 1992), the type of data analyzed (PG: photographic, VIS: visual telescopic observations, $\mathrm{B}$ : photoelectric $B$, and $\mathrm{V}$ : photoelectric $V$ ), the number of observations used to establish the observed light maxima, and the source of the observations, in addition to the temporal parameters. The data are plotted in Figure 10.

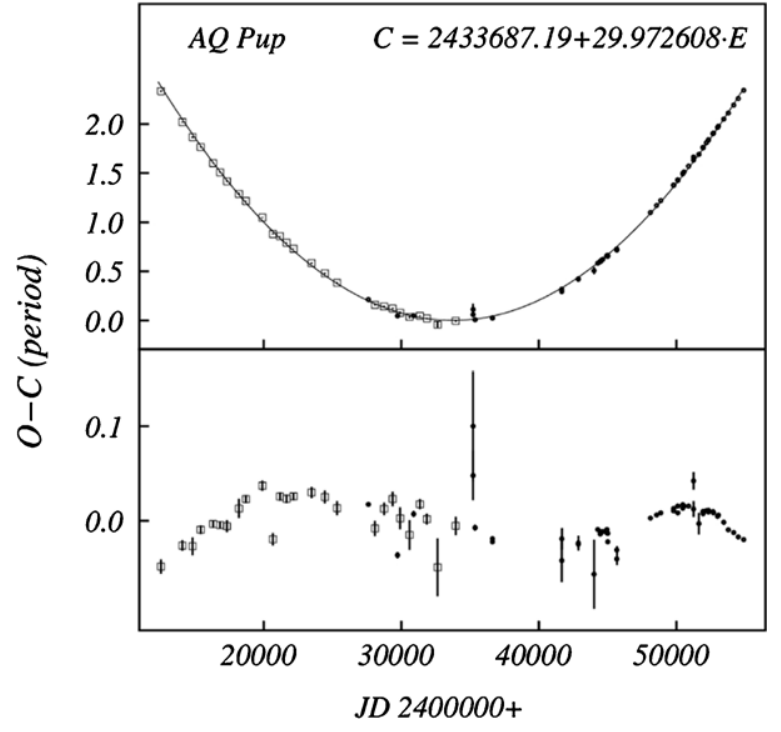

Figure 10. Differences between observed $(\mathrm{O})$ and computed $(\mathrm{C})$ times of light maximum for AQ Pup, computed in units of pulsation phase. Data based on photoelectric observations are denoted as filled circles and others as open circles. The upper diagram shows the actual $\mathrm{O}-\mathrm{C}$ variations with their uncertainties, the lower diagram the residuals from the calculated parabolic evolutionary trend.

A regression analysis of the $\mathrm{O}-\mathrm{C}$ data of Table 3 produced a parabolic solution for the ephemeris defined by

$$
\begin{aligned}
\mathrm{JD}_{\max }= & 2433687.1920( \pm 0.1397) \\
& +29.9726( \pm 0.0002) E+142.49( \pm 0.56) \times 10^{-6} E^{2},
\end{aligned}
$$

which overlays the data in Figure 10. The parabolic trend corresponds to a rapid period increase of $+300.05 \pm 1.18 \mathrm{~s} \mathrm{yr}^{-1}$, a value representative of the most rapid rate of period increase for Cepheids of comparable pulsation period.

In their study of Cepheid period changes, Turner et al. (2006) noted that the observed rates of period change were consistent with predictions from evolutionary models for stars in the first, second (period decreases), and third crossing of the instability strip, despite a wide range in predicted rates. The observed rates for the luminous, long-period variables also exhibited a smaller dispersion than that derived from various published models, and it was speculated that the Cepheid sample was dominated by stars in the second and third crossings of the strip, which are the slowest. Some older models, such as those of Iben (1967) for example, predicted fourth and fifth crossings for stars in the thick helium-burning shell phase, which occurs at a more rapid rate than second and third crossings for stars of the same mass. Turner et al. (2006) therefore speculated that the main sample of Cepheids consisted of second and third crossers, with a smaller group of putative fourth and fifth crossers exhibiting period changes at rates an order of magnitude larger than those of other Cepheids of similar period. As indicated in Figure 11, AQ Pup falls in the latter category according to its observed rate of period increase. While the location of AQ Pup in Figure 9 relative to the model isochrone for $\log t=7.5$ is consistent with either a third or fifth crossing, further evidence would be useful.

A potential complication is an unknown rate of mass loss for AQ Pup. Neilson et al. (2012) have noted that enhanced mass loss in Cepheids can elevate the rate of period increase for Cepheids displaying positive period changes, implying that a rapid rate of mass loss might explain the high rate of period increase for AQ Pup relative to other Cepheids of similar period in the third crossing of the instability strip. The Cepheid has an 
Table 3

Times of Maximum Light for AQ Pup

\begin{tabular}{|c|c|c|c|c|c|c|}
\hline $\mathrm{HJD}_{\max }$ & $\pm \sigma$ & Band & $\begin{array}{l}\text { Epoch } \\
(E)\end{array}$ & $\begin{array}{c}\mathrm{O}-\mathrm{C} \\
\text { (days) }\end{array}$ & $\begin{array}{c}\text { Observations } \\
(n)\end{array}$ & Reference \\
\hline 2412536.650 & 0.228 & PG & -708 & 70.065 & 9 & This paper (Harvard) \\
\hline 2414085.787 & 0.168 & PG & -656 & 60.627 & 9 & This paper (Harvard) \\
\hline 2414830.494 & 0.286 & PG & -631 & 56.018 & 9 & This paper (Harvard) \\
\hline 2415426.928 & 0.134 & PG & -611 & 53.000 & 32 & This paper (Harvard) \\
\hline 2416321.197 & 0.115 & PG & -581 & 48.091 & 50 & This paper (Harvard) \\
\hline 2416827.920 & 0.107 & PG & -564 & 45.279 & 31 & This paper (Harvard) \\
\hline 2417334.729 & 0.190 & PG & -547 & 42.554 & 28 & This paper (Harvard) \\
\hline 2418200.094 & 0.306 & PG & -518 & 38.714 & 38 & This paper (Harvard) \\
\hline 2418707.457 & 0.128 & PG & -501 & 36.542 & 27 & This paper (Harvard) \\
\hline 2419901.302 & 0.165 & PG & -461 & 31.483 & 28 & This paper (Harvard) \\
\hline 2420675.573 & 0.198 & $\mathrm{PG}$ & -435 & 26.466 & 26 & This paper (Harvard) \\
\hline 2421184.394 & 0.134 & PG & -418 & 25.753 & 49 & This paper (Harvard) \\
\hline 2421662.015 & 0.135 & PG & -402 & 23.812 & 30 & This paper (Harvard) \\
\hline 2422169.723 & 0.123 & PG & -385 & 21.986 & 30 & This paper (Harvard) \\
\hline 2423484.089 & 0.181 & PG & -341 & 17.556 & 30 & This paper (Harvard) \\
\hline 2424440.107 & 0.203 & PG & -309 & 14.451 & 24 & This paper (Harvard) \\
\hline 2425336.419 & 0.224 & PG & -279 & 11.585 & 18 & This paper (Harvard) \\
\hline 2427609.234 & 0.047 & PG & -203 & 6.482 & 30 & O’Leary (1936) \\
\hline 2428087.145 & 0.244 & PG & -187 & 4.831 & 32 & This paper (Harvard) \\
\hline 2428746.059 & 0.204 & PG & -165 & 4.348 & 32 & This paper (Harvard) \\
\hline 2429374.870 & 0.231 & PG & -144 & 3.733 & 26 & This paper (Harvard) \\
\hline 2429732.290 & 0.102 & PG & -132 & 1.482 & 30 & Charlier (1965) \\
\hline 2429913.074 & 0.346 & $\mathrm{PG}$ & -126 & 2.431 & 34 & This paper (Harvard) \\
\hline 2430601.166 & 0.470 & PG & -103 & 1.153 & 39 & This paper (Harvard) \\
\hline 2430901.277 & 0.098 & PG & -93 & 1.538 & 16 & Erleksova (1965) \\
\hline 2431350.810 & 0.163 & PG & -78 & 1.481 & 33 & This paper (Harvard) \\
\hline 2431859.537 & 0.173 & PG & -61 & 0.674 & 26 & This paper (Harvard) \\
\hline 2432636.941 & 0.913 & $\mathrm{PG}$ & -35 & -1.209 & 27 & This paper (Harvard) \\
\hline 2433956.885 & 0.289 & PG & +9 & -0.061 & 35 & This paper (Harvard) \\
\hline 2435217.689 & 0.780 & $B$ & +51 & 1.894 & 8 & Irwin (1961) \\
\hline 2435219.162 & 1.733 & $V$ & +51 & 3.367 & 8 & Irwin (1961) \\
\hline 2435365.976 & 0.094 & PG & +56 & 0.318 & 17 & Erleksova (1965) \\
\hline 2436625.298 & 0.035 & $B$ & +98 & 0.791 & 14 & Fernie et al. (1966) \\
\hline 2436625.308 & 0.027 & $V$ & +98 & 0.800 & 16 & Fernie et al. (1966) \\
\hline 2441668.729 & 0.685 & V & +266 & 8.823 & 9 & Madore (1975) \\
\hline 2441669.510 & 0.334 & $B$ & +266 & 9.604 & 9 & Madore (1975) \\
\hline 2442871.448 & 0.235 & V & +306 & 12.637 & 8 & Dean (1977) \\
\hline 2442871.503 & 0.137 & $B$ & +306 & 12.693 & 8 & Dean (1977) \\
\hline 2444012.939 & 1.096 & $V$ & +344 & 15.170 & 5 & Harris (1980) \\
\hline 2444285.002 & 0.045 & $V$ & +353 & 17.480 & 12 & Eggen (1983) \\
\hline 2444465.335 & 0.027 & $V$ & +359 & 17.976 & 13 & Coulson \& Caldwell (1985) \\
\hline 2444465.395 & 0.023 & $B$ & +359 & 18.036 & 12 & Coulson \& Caldwell (1985) \\
\hline 2444525.621 & 0.041 & $B$ & +361 & 18.317 & 33 & Eggen (1983) \\
\hline 2444645.837 & 0.051 & V & +365 & 18.643 & 21 & Eggen (1983) \\
\hline 2444946.666 & 0.021 & $B$ & +375 & 19.746 & 10 & Coulson \& Caldwell (1985) \\
\hline 2444946.681 & 0.020 & $V$ & +375 & 19.761 & 10 & Coulson \& Caldwell (1985) \\
\hline 2445006.453 & 0.038 & $V$ & +377 & 19.587 & 29 & Moffett \& Barnes (1984) \\
\hline 2445006.810 & 0.018 & $B$ & +377 & 19.944 & 28 & Moffett \& Barnes (1984) \\
\hline 2445667.740 & 0.194 & $V$ & +399 & 21.477 & 7 & Berdnikov (1986) \\
\hline 2445668.115 & 0.122 & $B$ & +399 & 21.852 & 6 & Berdnikov (1986) \\
\hline 2448106.960 & 0.021 & $V$ & +480 & 32.916 & 79 & ESA (1997) \\
\hline 2448558.731 & 0.028 & $V$ & +495 & 35.098 & 75 & ESA (1997) \\
\hline 2448859.950 & 0.032 & $V$ & +505 & 36.590 & 27 & ESA (1997) \\
\hline 2449793.836 & 0.020 & $V$ & +536 & 41.325 & 25 & Berdnikov \& Turner (1995) \\
\hline 2449793.855 & 0.014 & $B$ & +536 & 41.344 & 24 & Berdnikov \& Turner (1995) \\
\hline 2450095.059 & 0.028 & $B$ & +546 & 42.822 & 10 & Bersier (2002) \\
\hline 2450095.182 & 0.033 & $V$ & +546 & 42.946 & 10 & Bersier (2002) \\
\hline 2450456.767 & 0.020 & $B$ & +558 & 44.859 & 18 & Bersier (2002) \\
\hline 2450456.785 & 0.010 & $V$ & +558 & 44.877 & 17 & Bersier (2002) \\
\hline 2450547.137 & 0.011 & $V$ & +561 & 45.311 & 20 & Berdnikov \& Turner (1998) \\
\hline 2450878.613 & 0.015 & $V$ & +572 & 47.088 & 33 & Berdnikov \& Turner (2000) \\
\hline 2451240.167 & 0.253 & $V$ & +584 & 48.971 & 35 & Berdnikov \& Turner (2001a) \\
\hline 2451241.151 & 0.279 & $B$ & +584 & 49.955 & 35 & Berdnikov \& Turner (2001a) \\
\hline 2451631.535 & 0.341 & V & +597 & 50.695 & 23 & Berdnikov \& Caldwell (2001) \\
\hline
\end{tabular}


Table 3

(Continued)

\begin{tabular}{lcccccc}
\hline \hline HJD $_{\max }$ & $\pm \sigma$ & Band & $\begin{array}{c}\text { Epoch } \\
(E)\end{array}$ & $\begin{array}{c}\text { O-C } \\
(\text { days })\end{array}$ & $\begin{array}{c}\text { Observations } \\
(n)\end{array}$ & Reference \\
\hline 2451933.341 & 0.013 & $V$ & +607 & 52.776 & 66 & Pojmanski (2002) \\
2451933.364 & 0.031 & $V$ & +607 & 52.798 & 17 & Berdnikov \& Turner (2001b) \\
2451933.385 & 0.022 & $B$ & +607 & 52.819 & 17 & Berdnikov \& Turner (2001b) \\
2452174.563 & 0.031 & $V$ & +615 & 54.216 & 36 & Pojmanski (2002) \\
2452325.317 & 0.016 & $V$ & +620 & 55.107 & 38 & Berdnikov \& Turner (2004a) \\
2452325.355 & 0.018 & $B$ & +620 & 55.145 & 37 & Berdnikov \& Turner (2004a) \\
2452656.932 & 0.013 & $V$ & +631 & 57.024 & 42 & Berdnikov \& Turner (2004b) \\
2452687.050 & 0.011 & $V$ & +632 & 57.169 & 83 & Pojmanski (2002) \\
2452988.481 & 0.024 & $V$ & +642 & 58.874 & 32 & Berdnikov \& Turner (2004c) \\
2453048.828 & 0.010 & $V$ & +644 & 59.276 & 86 & Pojmanski (2002) \\
2453440.655 & 0.016 & $V$ & +657 & 61.458 & 64 & Pojmanski (2002) \\
2453772.192 & 0.013 & $V$ & +668 & 63.297 & 97 & Pojmanski (2002) \\
2454164.248 & 0.019 & $V$ & +681 & 65.710 & 61 & Pojmanski (2002) \\
2454495.959 & 0.017 & $V$ & +692 & 67.722 & 107 & Pojmanski (2002) \\
2454888.110 & 0.016 & $V$ & +705 & 70.228 & 98 & Pojmanski (2002) \\
\hline
\end{tabular}

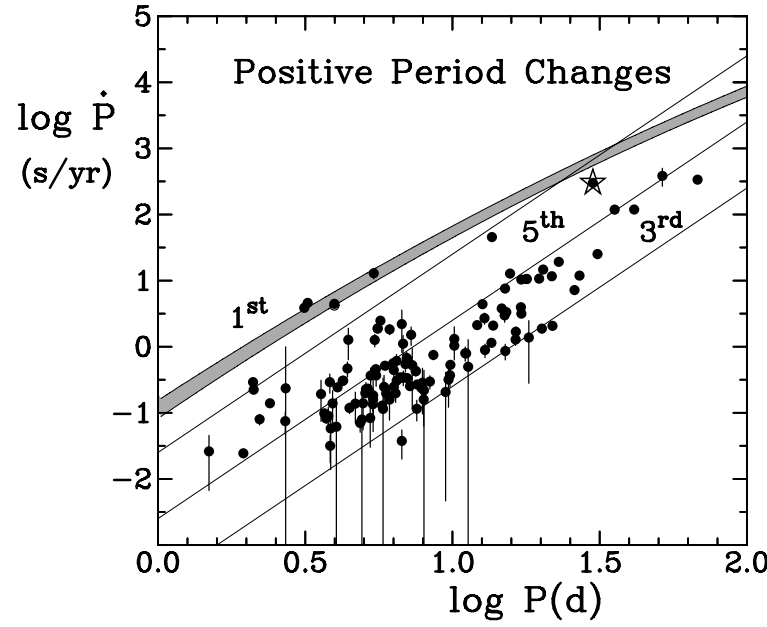

Figure 11. Observed and predicted rates of period change for Cepheids exhibiting period increases, with lines used to denote stars with period increases consistent with a third crossing of the instability strip, a separate group designated as putative fifth crossers, and a gray band representing predictions for first-crossing Cepheids. The rate for AQ Pup is denoted by a star symbol.

atmospheric composition, $[\mathrm{Fe} / \mathrm{H}]=-0.08 \pm 0.01$ (Romaniello et al. 2008), indicating slightly less than solar metallicity. A more detailed spectroscopic study centered on abundances of the CNO elements, to test for dredge-up material for example, is not necessarily useful for establishing evolutionary status (Turner \& Berdnikov 2004) because of possible meridional mixing during the main-sequence stage (Maeder 2001). A search for features indicative of mass loss would be more informative.

The residuals from the parabolic fit to the $\mathrm{O}-\mathrm{C}$ data are plotted in the lower part of Figure 10. They do not appear to be randomly distributed, displaying instead a sinusoidal trend suggesting the possibility of light travel time effects in a binary system. Unfortunately, the implied orbital period of $\sim 84$ years and semi-major axis of $\sim 1.2$ lt-day produce an uncomfortably large minimum total mass for the co-orbiting stars, so such a possibility must be tested by other means, from radial velocity measures for example. An Eddington-Plakidis test (Eddington $\&$ Plakidis 1929) on the residuals shown in Figure 12 also displays no evidence for random fluctuations in period (see Turner et al. 2009b). Chaotic period fluctuations in AQ Pup would normally be revealed by an upward slope to the plotted

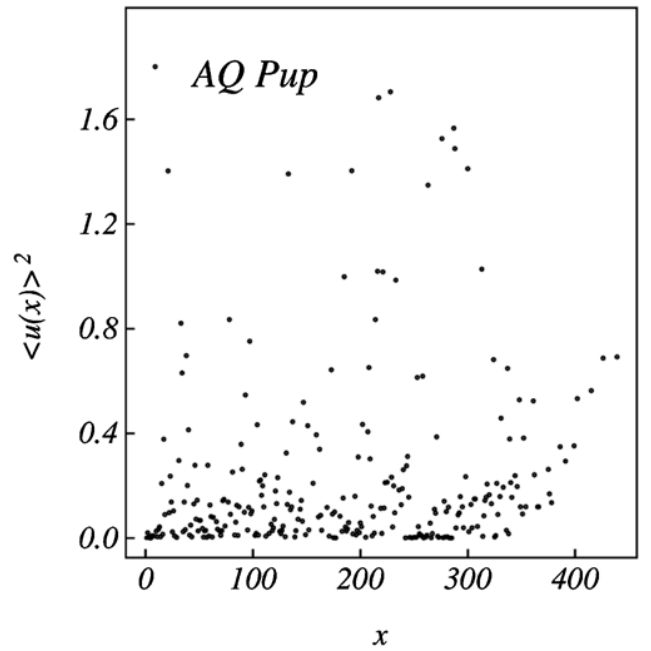

Figure 12. Eddington-Plakidis test of the residuals from the observed times of light maximum for AQ Pup displays no evidence for random fluctuations in period. If that were the case, the data would scatter about a positive slope rather than the null slope as indicated.

Table 4

Derived Parameters for AQ Pup and V620 Pup

\begin{tabular}{lcc}
\hline \hline Parameter & AQ Pup & \\
\hline $\log P$ & 1.4767 & V620 Pup \\
$\log \dot{P}\left(\mathrm{~s} \mathrm{yr}^{-1}\right)$ & $2.4772 \pm 0.0017$ & 0.4126 \\
$E_{B-V}$ & $0.47 \pm 0.07$ & $\ldots$ \\
$(\langle B\rangle-\langle V\rangle)_{0}$ & +0.89 & $0.64 \pm 0.02$ \\
$\left\langle M_{V}\right\rangle$ & $-5.40 \pm 0.25$ & +0.52 \\
$\log t(\mathrm{yr})$ & 7.5 & $-2.74 \pm 0.11$ \\
$d(\mathrm{pc})$ & $3213 \pm 185$ & 8.0 \\
\hline
\end{tabular}

data. Unless the tabulated individual times of light maximum are affected by temporal averaging (see Turner et al. 2009b), there is no evidence to suggest that the residuals of Figure 10 arise from random fluctuations in period. Perhaps they are indicative of episodic variations in the rate of mass loss in AQ Pup, which additional observations could test.

The inferred parameters for the two Cepheids are summarized in Table 4. The derived space reddening for AQ Pup compares well with a $B V I$ reddening of $E_{B-V}=0.504$ derived by 


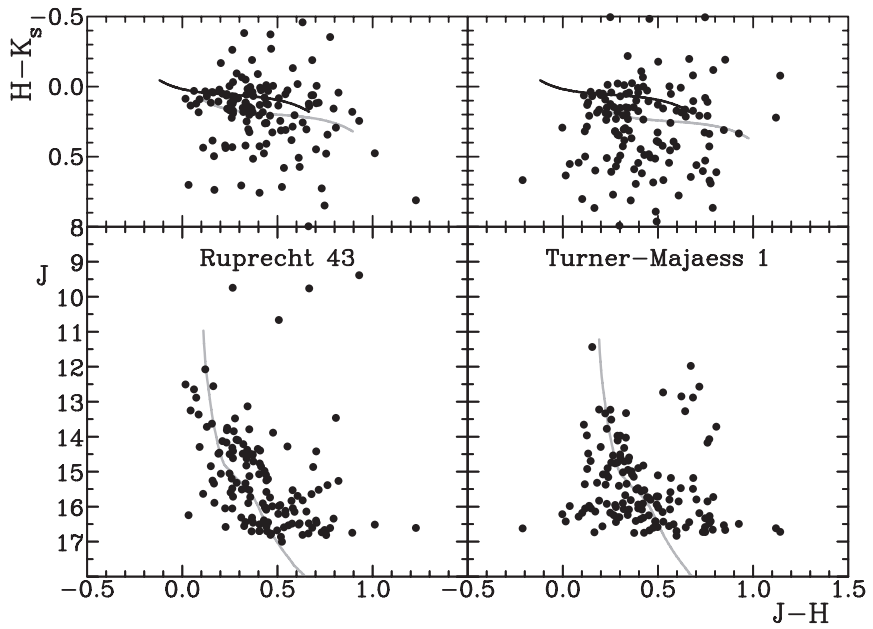

Figure 13. 2MASS color-color (upper) and color-magnitude (lower) diagrams for measured stars in Ruprecht 43 (left) and Turner-Majaess 1 (right). The intrinsic color-color relation is plotted as a black curve, while gray curves represent the same relation for reddenings of $E_{B-V}=0.68$ (left) and $E_{B-V}=$ 0.92 (right). The plotted ZAMS relations (gray curves, lower) correspond to the same reddenings for the intrinsic distance modulus of Figure 7.

Laney \& Caldwell (2007) and a model atmosphere reddening of $E_{B-V}=0.453$ found by Kovtyukh et al. (2008). The inferred distance of $3.21 \pm 0.19 \mathrm{kpc}$ to AQ Pup found here also agrees exactly with the estimate of $3.21 \mathrm{kpc}$ derived by Storm et al. (2011) using the infrared surface brightness version of the Baade-Wesselink method. However, the values of $E_{B-V}=0.518$ and $\left\langle M_{V}\right\rangle=-5.51$ inferred for AQ Pup by Storm et al. (2011) differ slightly from the present results. The implied luminosity of AQ Pup as a member of Turner 14 is $\left\langle M_{V}\right\rangle=-5.40 \pm 0.25$ with the uncertainty in reddening included. The difference relative to the Storm et al. (2011) results is small, and the parameters are consistent with a Cepheid lying near the center of the instability strip, as implied by its relatively large light amplitude of $\Delta B=1.84$. The agreement might be optimized if AQ Pup were in a putative fifth crossing lying slightly toward the cool edge of the instability strip. The derived luminosity of AQ Pup is otherwise exactly that predicted by the period-radius and color-effective temperature relations of Turner \& Burke (2002), providing further confirmation of their validity.

The photometric parallax of $\pi=0.31 \pm 0.02$ mas derived here for AQ Pup can be compared with parallaxes from the Hipparcos mission of $8.85 \pm 4.03$ mas (ESA 1997) and $15.47 \pm 3.59$ mas (van Leeuwen 2007), a difference of more than $2 \sigma$ for the original Hipparcos estimate and more than $4 \sigma$ for the revised value. Conceivably, the discrepancy arises from contamination by the nearby companions to AQ Pup, as suggested by Szabados (1997) for other Cepheids observed by Hipparcos.

V620 Pup is not studied well enough to provide a comparison with previous studies. The implied space reddening yields an intrinsic $(\langle B\rangle-\langle V\rangle)_{0}$ color of +0.52 and a luminosity as a member of Turner 13 of $\left\langle M_{V}\right\rangle=-2.74 \pm 0.11$. The luminosity is $\sim 0.40$ mag more luminous than would be predicted by the relations of Turner \& Burke (2002) for a 2.59 classical Cepheid, but that could be explained if the Cepheid lies on the blue side of the instability strip (as for the case of a first crossing) or is an overtone pulsator. The derived intrinsic color appears to indicate a Cepheid lying near strip center, or blueward of strip center for the case of overtone pulsation. The implied isochrone fit for Turner 13 in Figure 8 indicates that V620 Pup is in the first crossing, which, if true, would be accompanied by measurable increases in pulsation period over a short time interval. The Cepheid has not been observed long enough to test such a possibility, but archival images may contain information for investigating that further.

A 12 th magnitude star $\sim 0.5$ southeast of V620 Pup appears to be a reddened F-type star, possibly a giant. It is the object 0.86 mag more luminous than the Cepheid in Figure 8. The photometric analysis suggests that it may also lie close to the Cepheid instability strip, provided it is an evolved cluster member. However, there is no indication of variability in the star from photometric monitoring of the V620 Pup field.

\section{CONCLUSIONS}

Although there are no obvious OB associations in the region, a $U B V$ survey of a field located around the Cepheids AQ Pup and V620 Pup reveals the presence of two putative clusters: one centered near V620 Pup that appears to contain the Cepheid as a likely member, and the other centered $\sim 17^{\prime}$ south of AQ Pup, with outlying stars surrounding the Cepheid, that appears to contain the 29.97 pulsator as a member. Both clusters are faint, poorly populated, and near the limits of imaging surveys like the Palomar and ESO-SRC atlases. Their existence is argued by the dereddened parameters of likely cluster members, including the Cepheids.

The possible association of AQ Pup with the cluster Ruprecht 43 suggested by Tsarevsky et al. (1966) has never been investigated fully, primarily because the coordinates cited for Ruprecht 43 appear to be in error. There is a star chain centered on J2000.0 coordinates $07: 58: 46,-28: 48: 47$, that has the appearance of a small, faint, compact cluster, and the declination differs by only $10^{\prime}$ from the value cited for Ruprecht 43 by B. S. Alessi (2012, private communication). The original coordinates cited for this cluster may be incorrect. There is also an overlooked faint cluster of stars located closer to AQ Pup at J2000.0 coordinates 07:59:19, -28:58:00, designated here as Turner-Majaess 1. Ruprecht 43 and Turner-Majaess 1 both lie outside of the area surveyed in Figure 1.

2MASS color-color and color-magnitude diagrams for stars lying within $2^{\prime}$ of the adopted centers for the two clusters are presented in Figure 13. The deduced reddenings for the two clusters, $E_{B-V}=0.68$ and 0.92 , respectively, were inferred using the techniques outlined by Turner (2011), while the distance moduli were chosen to yield intrinsic values identical to the best-fitting results of Figure 7, which apply to AQ Pup and Turner 14. They were not derived using best-fitting procedures, although it is noteworthy that the data for both clusters are a reasonably good match to the adopted values. Such good agreement argues that Ruprecht 43 and Turner-Majaess 1 probably lie at roughly the same distance as AQ Pup and Turner 14, suggesting that they belong to the same star complex. Deeper photometry is required to explore that conclusion further.

Absolute magnitudes have been derived for AQ Pup and V620 Pup under the assumption that they are members of the clusters in their vicinity. The case for AQ Pup seems reasonably strong, and the Cepheid appears to be in the third or possibly fifth crossing of the instability strip. Its rapid rate of period increase might also be indicative of rapid mass loss. V620 Pup is curious, given that the cluster match implies it is in the first crossing of the instability strip. However, it has completely different characteristics from other putative first crossers (Turner 2009), 
with a very skewed light curve and a sizeable light amplitude of $\Delta B=0.77$, more like that of second or third crossers. Further study of the Cepheid and the cluster surrounding it appears to be essential.

David Pass and Mark Starzomski made star counts for the field of AQ Pup as part of a research experience project while students at Prince Edward High School in Dartmouth, Nova Scotia. The present study was supported by the National Research Council of Canada and by research funding awarded through the Natural Sciences and Engineering Research Council of Canada (NSERC), and the Russian Foundation for Basic Research (RFBR). We are indebted to the director of Harvard College Observatory for access to the plate stacks, to Noel Carboni for supplying color images of the target fields, and to the referee for useful suggestions. This publication makes use of American Association of Variable Star Observers facilities as well as data products of the Two Micron All Sky Survey, which is a joint project of the University of Massachusetts and the Infrared Processing and Analysis Center/California Institute of Technology, funded by the National Aeronautics and Space Administration and the National Science Foundation.

\section{REFERENCES}

Berdnikov, L. N. 1986, Perem. Zvezdy, 22, 369

Berdnikov, L. N. 1992, Sov. Astron. Lett., 18, 207

Berdnikov, L. N., \& Caldwell, J. A. R. 2001, J. Astron. Data, 7, No. 3

Berdnikov, L. N., \& Turner, D. G. 1995, Astron. Lett., 21, 717

Berdnikov, L. N., \& Turner, D. G. 1998, Astron. Astrophys. Trans., 16, 291

Berdnikov, L. N., \& Turner, D. G. 2000, Astron. Astrophys. Trans., 18, 679

Berdnikov, L. N., \& Turner, D. G. 2001a, Astron. Astrophys. Trans., 19, 689

Berdnikov, L. N., \& Turner, D. G. 2001b, ApJS, 137, 209

Berdnikov, L. N., \& Turner, D. G. 2004a, Astron. Astrophys. Trans., 23, 253

Berdnikov, L. N., \& Turner, D. G. 2004b, Astron. Astrophys. Trans., 23, 395

Berdnikov, L. N., \& Turner, D. G. 2004c, Astron. Astrophys. Trans., 23, 599

Bersier, D. 2002, ApJS, 140, 465

Charlier, L. 1965, AnLei, 22, 147

Coulson, I. M., \& Caldwell, J. A. R. 1985, SAAOC, No., 9, 5

Cutri, R. M., Skrutskie, M. F., van Dyk, S., et al. 2003, The IRSA 2MASS AllSky Point Source Catalog of Point Sources, NASA/IPAC Infrared Science Archive

Dean, J. F. 1977, Mon. Notes Astron. Soc. South. Afr., 36, 3

Dias, W. S., Alessi, B. S., Moitinho, A., \& Lépine, J. R. D. 2002, A\&A, 389, 871

Eddington, A. S., \& Plakidis, S. 1929, MNRAS, 90, 65

Eggen, O. J. 1983, AJ, 88, 998

Erleksova, G. E. 1965, Perem. Zvezdy, 15, 590

ESA 1977, The Hipparcos and Tycho Catalogues, European Space AgencySP1200 (Lisse: ESA)

Evans, N. R., \& Udalski, A. 1994, AJ, 108, 653

Fernie, J. D. 1963, AJ, 68, 780

Fernie, J. D., Hiltner, W. A., \& Kraft, R. P. 1966, AJ, 71, 999

Grubissich, C. 1968, Z. Astrophys., 68, 176

Harris, H. C. 1980, PhD thesis, Univ. Washington

Havlen, R. J. 1972, A\&A, 17, 413

Iben, I., Jr. 1967, ARA\&A, 5, 571

Irwin, J. B. 1955, Mon. Notes Astron. Soc. South. Afr., 14, 38

Irwin, J. B. 1958, AJ, 63, 197

Irwin, J. B. 1961, ApJS, 6, 253

Kazarovets, E. V., Samus, N. N., Durlevich, O. V., Kireeva, N. N., \& Pastukhova, E. N. 2008, IBVS, 5863, 1
Kharchenko, N. V., \& Roeser, S. 2001, Kinematika Fiz. Nebesnykh Tel, 17, 409 Kholopov, P. N. 1956, Perem. Zvezdy, 11, 325

Kovtyukh, V. V., Soubiran, C., Luck, R. E., et al. 2008, MNRAS, 389, 1336

Kraft, R. P. 1957, ApJ, 126, 225

Laney, C. D., \& Caldwell, J. A. R. 2007, MNRAS, 377, 147

Madore, B. F. 1975, ApJS, 29, 219

Maeder, A. 2001, Ap\&SS, 277, 291

Massey, P. 2002, ApJS, 141, 81

Meynet, G., Mermilliod, J.-C., \& Maeder, A. 1993, A\&AS, 98, 47

Moffett, T. J., \& Barnes, T. G. 1984, ApJS, 55, 389

Neckel, Th., \& Klare, G. 1980, A\&AS, 42, 251

Neilson, H. R., Engle, S. G., Guinan, E., et al. 2012, ApJ, 745, L32

O’Leary, S. J. 1936, PRCO, 2, 17

Pietrukowicz, P. 2003, Acta Astron., 53, 63

Pojmanski, G. 2002, Acta Astron., 52, 397

Romaniello, M., Primas, F., Mottini, M., et al. 2008, A\&A, 488, 731

Shorlin, S. L., Turner, D. G., \& Pedreros, M. H. 2004, PASP, 116, 170

Stephenson, C. B., \& Sanduleak, N. 1971, Luminous Stars in the Couthern Milky Way, PW\&SO, Vol. 1, No. 1 (Cleveland, OH: Warner and Swasey Observatory)

Storm, J., Gieren, W., Fouqué, P., et al. 2011, A\&A, 534, A94

Szabados, L. 1997, in Proc. ESA Symposium "Hipparcos-Venice '97," ed. B. Battrick, M. A. C. Perryman, \& P. L. Bernacca (ESA SP-402) (Noordwijk: ESA), 657

Tifft, W. G. 1959, ApJ, 129, 241

Tsarevsky, G. S. 1970, Nauchnye Inf., 16, 117

Tsarevsky, G. S., Ureche, V., \& Efremov, Y. N. 1966, ATsir, 367, 1

Turner, D. G. 1976a, AJ, 81, 97

Turner, D. G. 1976b, AJ, 81, 1125

Turner, D. G. 1979, PASP, 91, 642

Turner, D. G. 1981, AJ, 86, 222

Turner, D. G. 1989, AJ, 98, 2300

Turner, D. G. 1993, A\&A, 97, 755

Turner, D. G. 1994, RevMexAA, 29, 163

Turner, D. G. 1996, in ASP Conf. Ser. 90, The Origins, Evolution, and Destinies of Binary Stars in Clusters, ed. E. F. Milone \& J.-C. Mermilliod (San Francisco, CA: ASP), 443

Turner, D. G. 2009, in AIP Conf. Ser. 1170, Stellar Pulsation: Challenges for Theory and Observation, ed. J. A. Guzik \& P. A. Bradley (Melville, NY: AIP), 59

Turner, D. G. 2010, Ap\&SS, 326, 219

Turner, D. G. 2011, RevMexAA, 47, 127

Turner, D. G., \& Berdnikov, L. N. 2004, A\&A, 423, 335

Turner, D. G., Berdnikov, L. N., \& Abdel-Sabour Abdel-Latif, M. 2006, PASP, 118,410

Turner, D. G., \& Burke, J. F. 2002, AJ, 124, 2931

Turner, D. G., Forbes, D., Leonard, P. J. T., et al. 2009a, MNRAS, 397, 1046

Turner, D. G., Forbes, D., \& Pedreros, M. 1992, AJ, 104, 1132

Turner, D. G., Forbes, D., van den Bergh, S., Younger, P. F., \& Berdnikov, L. N. 2005, AJ, 130, 1194

Turner, D. G., Percy, J. R., Colivas, T., Berdnikov, L. N., \& Abdel-Sabour AbdelLatif, M. 2009b, in AIP Conf. Ser. 1170, Stellar Pulsation: Challenges for Theory and Observation, ed. J. A. Guzik \& P. A. Bradley (Melville, NY: AIP), 167

Turner, D. G., van den Bergh, S., Younger, P. F., Danks, T. A., \& Forbes, D. 2010, ApJS, 85, 119

Turner, D. G., \& Welch, G. A. 1989, PASP, 101, 1038

van den Bergh, S. 1957, ApJ, 126, 323

van den Bergh, S., Brosterhus, E. B. F., \& Alcaino, G. 1982, ApJS, 50,529

van den Bergh, S., Younger, P. F., Brosterhus, E. B. F., \& Alcaino, G. 1983, ApJS, 53,765

van den Bergh, S., Younger, P. F., \& Turner, D. G. 1985, ApJS, 57, 743

van Leeuwen, F. 2007, A\&A, 474, 653

Vehrenberg, H. 1964, Photographischer Stern-Atlas Falkauer Atlas Südhimmel (Düsseldorf: Treugesell-Verlag)

Zacharias, N., Monet, D., Levine, S., et al. 2005, BAAS, 36, 1418 